\title{
Managing acute collapse in pregnant women
}

\author{
Hennie Lombaard, Doctor*, Priya Soma-Pillay, Doctor, \\ El-Marie Farrell, Doctor
}

Department of Obstetrics and Gynecology, University of Pretoria, Pretoria, South Africa

\section{Keywords:}

acute collapse

pulmonary embolism

amniotic fluid embolism

acute coronary syndrome

mechanical prosthetic heart valves

acute aortic dissection

cerebrovascular incidents and anaesthetic

complications

failed intubation

anaphylaxis

\begin{abstract}
The most important causes of acute collapse in pregnancy are pulmonary embolism, amniotic fluid embolism, acute coronary syndrome, thrombosed mechanical prosthetic heart valves, acute aortic dissection, cerebrovascular incidents and anaesthetic complications like failed intubation, anaphylaxis, and problems relating to regional or local anaesthetic agents. The management is based on supporting the different organ systems that are affected. The diagnosis of pulmonary embolism is based on a clinical suspicion supported by certain diagnostic test. Tests like D-dimers have their limitations and cannot be used alone to exclude the diagnosis especially when there is a high clinical suspicion. The choice of the best diagnostic tool is based upon weighing longterm risks to both mother and foetus on the one side and delaying the diagnosis on the other side. The management of acute coronary syndrome is based on immediate angiography and percutaneous coronary intervention. Although there are reports of the use of clopidrogel in pregnancy, there are few data on its effect on the foetus. There is no clinical evidence for fibrinolytic therapy as a reperfusion strategy in pregnancy and it is best avoided as the risk of haemorrhage outweighs the possible benefit of treatment. Patients with a prosthetic heart valve that present with a disappearance of the prosthetic heart sounds or a new murmur should get an urgent cardiac ultrasound to rule out a thrombosed prosthetic valve. Anaesthesia-related causes are an increasing cause of maternal morbidity and mortality.
\end{abstract}

() 2009 Elsevier Ltd. All rights reserved.

\footnotetext{
* Corresponding author. Tel.: +27834078632, 27123542849 (Work); Fax: +27123296258.

E-mail address: hennie.lombaard@up.ac.za (H. Lombaard).
} 


\section{Introduction}

Six percent of direct maternal deaths in South Africa are due to acute collapse. ${ }^{1}$ The most important causes of acute collapse in pregnancy are pulmonary embolism, amniotic fluid embolism, acute coronary syndrome, thrombosed mechanical prosthetic heart valves, acute aortic dissection, cerebrovascular incidents and anaesthetic complications like failed intubation, anaphylaxis, and problems relating to regional or local anaesthetic agents.

In the 2003-2005 Why Mothers Die Report, thromboembolism and amniotic fluid embolism were responsible for $43.9 \%$ of direct deaths, while cardiac disease in pregnancy was the leading cause of maternal deaths overall. ${ }^{2}$ The most common causes of cardiac disease in this report were myocardial infarction and aortic dissection. ${ }^{2}$ There were 85 maternal deaths complicated by cardiac disease in SA between 2002 and $2004{ }^{1}$

Although the incidence of acute collapse directly related to anaesthesia is relatively rare in the developed world, the use of anaesthetic agents in the labouring patient is continuously rising. This not only relates to the ever-rising incidence of caesarean sections, but also to the extensive use of regional blocks, i.e. epidural anaesthesia, in the patients who still attempt to deliver vaginally. It is therefore becoming more important to pay careful attention to complications, especially life-threatening complications, which may arise due to the use of these agents.

Most of the conditions discussed in this chapter are rare and management protocols are lacking evidence from randomised controlled trials, so case studies and expert opinion will be used to base recommendations on. Data from studies on nonpregnant patients will be extrapolated to the pregnant population. Diabetic keto-acidosis and thyroid storm will not be dealt with in this publication as they were discussed in an earlier publication. ${ }^{3}$

\section{Amniotic fluid embolism [AFE]}

This is a rare but lethal condition. The incidence of AFE is reported to be between 1:8,000 and $1: 80,000$ births. $^{4-9}$ The first report in 1926 by Meyer $^{8}$ suggested that there was occlusion of the pulmonary blood vessels by amniotic fluid or foetal cells. Clark et al. developed the modern concept of an anaphylactoid syndrome of pregnancy. ${ }^{5,8-10}$ The mortality of AFE varies from 61 to $86 \%$, 4,7 although a recent report found it as low as 16 to $37 \% ., 6,11$ The reported incidence varies as a cause of maternal death with $2.3 \%$ in the United Kingdom, $10 \%$ in the United States and 30\% in Singapore. ${ }^{5,7}$ Apart from the high mortality associated with amniotic fluid embolism, the incidence of neurological handicap is also significant with reports quoting figures between $7 \%{ }^{4}$ and $85 \%{ }^{7}$ Amniotic fluid embolism is usually associated with delivery but it can occur at any time during the pregnancy. ${ }^{4,5,8,12}$ It has been described with the following procedures: vacuum aspiration of the uterus in the first trimester, second trimester termination of pregnancy, amniocentesis, amnio-infusion and both vaginal and caesarean section delivery. ${ }^{4,5}$

\section{Clinical presentation ${ }^{4-7,12,13}$}

- Acute cardiovascular collapse

- Foetal compromise which can present as foetal bradycardia

- Acute respiratory failure or Adult Respiratory Distress Syndrome

- Cyanosis

- Hypotension

- Coagulopathy

The above were reported in more than $80 \%$ of patients presenting with AFE. ${ }^{4}$ The other symptoms and signs are ${ }^{4-7,12-15}$ :

- Dyspnoea

- Seizures

- Dysrythmia 
- Acute confusional state

- Atony

- Bronchospasm

- Transient hypertension

- Cough

- Headache

- Chest pain

- Decreased urine output

- Nausea and vomiting

- Fever and chills

- Maternal death

\section{Diagnosis}

Since patients with AFE present with any combination of the above signs and symptoms, making the diagnosis can be a challenge. This is further complicated by the fact that there is no single diagnostic test available. The differential diagnosis will include the following: ${ }^{4,5,12,16}$ :

- Obstetric-related haemorrhage causing haemovoleamic shock, including uterine rupture

- Cardiogenic shock

- Massive thromboembolism

- Septic shock

- Eclampsia

- High spinal

- Allergic reaction

- Incorrect drug administration

- Drug side effect

- Transfusion reaction

- Peripartum cardiomypathy

- Aspiration

Current research is evaluating the value of monoclonal antibody essay to the mucin-like glycoprotein sialyl Tn [STN] and immuno-histochemical staining with monoclonal antibody TKH-2, both of which have been found in higher concentration in women with suspected AFE, ${ }^{4,13,16-18}$ but at present the role of these tests is uncertain. ${ }^{13}$ The important tests in evaluating AFE should include the following ${ }^{4,15-17}$ :

- Chest X-ray

- Electrocardiogram [ECG)

- Serum tryptase

- Full blood count and platelet count

- Coagulation profile

- Arterial blood gas

These tests assess the current biochemical and haematological status of the patient and help to exclude other possible causes. ${ }^{5}$

Trans-oesophageal echocardiography (TOE) has been used to evaluate right heart function. These changes fit in with the first part of the patophysiology of AFE, ${ }^{4,7,9,12}$ namely pulmonary hypertension and right ventricular overload.

The findings of TOE that are indicative of AFE are ${ }^{7,9}$ a discrepancy between the left and right ventricles due to right ventricular overload that results in changes of the interventricular septum and a D-shaped right ventricle on the short axis views. The left ventricle appears volume depleted with normal function. The pulmonary trunks are dilated and there are no other clinical conditions that can explain these findings. There is also an increase in the pulmonary artery pressure and tricuspid regurgitation. ${ }^{9}$ 
A definite diagnosis can be made by demonstrating squamous epithelial cells in the maternal circulation, but this is not practical in the clinical setting. If central lines are placed, aspirates can be used to search for squamous cells.

There is no specific diagnostic test and a high index of suspicion is necessary when a pregnant patient presents with any of the above signs and symptoms. Clark used four of the following clinical and laboratory criteria that could not be explained by other causes for inclusion in the national registry ${ }^{4,17,20 \text { : }}$

- Acute hypotension or cardiac arrest

- Acute hypoxia diagnosed by cyanosis, dyspnoea and/or respiratory arrest

- Coagulopathy or severe haemorrhage that cannot be explained by other causes

- Absence of other explanations for the clinical manifestations seen

- Onset during labour or within 30 minutes of delivery or surgical abortion

\section{Management}

The management of women with AFE must be multi-disciplinary and the patient should be managed in an intensive care unit.

The basis of the management of AFE is support of airway, tissue oxygenation, breathing and circulation. ${ }^{4-6,16,17}$ To achieve this there are certain goals of treatment. To maintain tissue oxygenation, $100 \%$ supplemental oxygen is indicated with intubation and ventilation to maintain positive end respiratory pressure and attempt to achieve a level of $\mathrm{PaO}_{2} \geq 60 \mathrm{mmHg}$ with the saturation above $90 \%$. The administration of diuretics is recommended. ${ }^{4-6,13,17,19}$

For circulatory support the Advanced Cardiac Life Support [ACLS] protocol should be initiated with the mother in the left lateral decubitus position. ${ }^{4,13,16}$ Colloids and crystalloids should be administered with inotropes such as dopamine, dobutamine or phenylephrine. The aim is to improve the circulation and maintain a urine output of more than $25 \mathrm{ml} /$ hour with the mean arterial pressure above 65 mmHg. ${ }^{12,13,16}$ The $\beta$-adrenergic effects of dopamine, epinephrine and norepinephrine improve cardiac function in addition to the $\alpha$-adrenergic effect of phenylephrine. ${ }^{4,12}$ Other treatment modalities include nitric oxide (a selective pulmonary vasodilator), cardiopulmonary bypass, extracorporeal membrane oxygenation and intra-aortic balloon counter pulsation. ${ }^{4,9,12,16}$ Delivery of the foetus improves the survival of the foetus and helps with the maternal cardiopulmonary resuscitation. ${ }^{4,16,17,19}$ Intact foetal survival is possible if the foetus is delivered within 5 minutes. Improvement in maternal outcome is possible if the foetus is delivered within 4 minutes of developing malignant arrhythmia via a perimortem caesarean section. ${ }^{4}$

In cases where the mother develops convulsions or an altered level of consciousness, her hypoxia needs to be corrected and anti-epileptic drugs can be used. ${ }^{13}$ Central venous pressure and pulmonary arterial lines are recommended in the fluid management of these patients. ${ }^{43,17}$

The third leg of the management is to correct the coagulopathy. This can be done using freshfrozen plasma, packed red blood cells and platelets. Cryoprecipitate is not the first line therapy but has a role in volume-overloaded patients ${ }^{12,16}$ and also contains fibronectin that assists the reticuloendothelial system with the filtration of antigenic and toxic substances. ${ }^{12,16}$ Recombinant factor VIIa may be useful to treat the DIC caused by AFE. ${ }^{4,12}$ Obstetric haemorrhage should be aggressively prevented by ${ }^{5,12}$ balloon tamponade of the uterus, and uterine artery embolisation ${ }^{4}$ may be of help.

Other treatment modalities such as high dose steroids (500 mg hydrocortisone sodium succinate every 6 hours until improvement), antithrombin III infusion, leukotrine inhibitors, inhaled prostacyclines, hemofiltration or exchange transfusion have been described anecdotally and are unlikely to be tested scientifically. ${ }^{10,12,13,16,17}$

\section{Pulmonary embolism (PE)}

Pulmonary embolism remains a leading cause of direct maternal deaths in the United Kingdom and South Africa. ${ }^{1,2,21,22}$ In the United States it is responsible for $19.6 \%$ of pregnancy-related deaths. ${ }^{23}$ In Japan, PE causes more deaths in the 20 to 39 year age group than any other age and there was a 6.5 fold increase in reported cases of PE in obstetrics and gynaecology from 1991 to $2000 .^{24}$ 


\section{Clinical presentation}

The signs and symptoms of PE are the following ${ }^{15,23,25-29}$ :

- Dyspnoea and tachypnoea - this combination is present in $90 \%$ of patients

- Pleuritic chest pain

- Tachycardia

- Pleural rub

- Syncope or near syncope

- Vomiting

- Palpitations

- Fever

- Wheezing

- Haemoptysis

- Crackles

- Cough

- Signs of pulmonary hypertension:

$\square$ Swollen neck veins

$\square$ Loud $\mathrm{P}_{2}$

$\square$ Right-sided gallop

$\square$ Right ventricular lift

The problem is that the signs and symptoms are non-specific..$^{23,26,28,30}$ Some reports found the combination of dyspnoea, tachypnoea, pleuritic chest pain and syncope or near syncope in $90 \%$ of cases $^{23,28}$ while other data showed that 60 to $80 \%$ of patients who died had no clinical signs or symptoms. ${ }^{27}$

\section{Diagnosis}

Diagnosis is based on a high index of suspicion and also a number of special investigations. Identifying women at risk of developing a pulmonary embolism and then having it as part of the differential diagnosis once a patient presents with any of the signs and symptoms is essential. The United Kingdom Obstetric Surveillance System (UKOSS) looked at a number of risk factors for pulmonary embolism but found only two with a significantly raised odds ratio $(\mathrm{OR})^{22}$ :

- Body Mass Index (BMI) greater than 30. Odds Ratio (OR) 2.65 with 95\% Confidence Interval (CI) of 1.09-6.45

- Multiparous women (OR 4.03, 95\% CI 1.60-9.84)

Routine investigations. Arterial blood gasses: most patients with $\mathrm{PE}$ will have a low $\mathrm{PaO}_{2}$ and raised $\mathrm{PaCO}_{2}$, but in up to $20 \%$ of women with PE this can be normal. ${ }^{23,25,27,29,30}$ The chest X-ray findings include: atelectasis, infiltrates, effusion, regional oligaemia and pulmonary oedema. ${ }^{23,27,31,32}$ The classical wedge shape infiltrate or decreased vascularity is seldom seen. The chest X-ray can be normal in up to $50 \%$ of patients but may help in excluding other pathology. ${ }^{25,27,30,32,33}$ The classical signs on ECG of a deep S wave in lead I, inverted T and Q waves in lead III and signs of right heart strain are infrequent ${ }^{25,27,32}$ and not of value in the diagnosis if there is reasonable clinical suspicion of PE. ${ }^{23,30,31}$

D-dimer testing has been used in the screening of patients with suspected pulmonary embolism. ${ }^{23,25,30-32} \mathrm{D}$-dimer testing is dependent on the type of testing method that is used and unfortunately different methods have different values and sensitivities. ${ }^{23,25}$ There is some controversy regarding the use after a case report described a patient with a normal D-dimers and pulmonary embolism. ${ }^{21} \mathrm{~A}$ study in nonpregnant patients found the sensitivity $97 \%$, the specificity $26.9 \%$, and the negative predictive value $77.7 \%$ in patients with a high clinical probability and for patients with a low clinical probability the sensitivity was $95.6 \%$, the specificity $40.5 \%$ and the negative predictive value $96.2 \%{ }^{34}$ There is a strong correlation between the location of the pulmonary embolism and the accuracy of D-dimers. The accuracy of D-dimers is higher with larger emboli or multiple segmental emboli than with subsegmental emboli. ${ }^{33}$ 
D-dimer testing should be used in relation with the clinical probability of the patient and a negative D-dimer is of value in a patient with a low probability, but should not be used in a patient with a high probability because of the low positive predictive value of $77 \%$ and can also be of value in patients less than 20 weeks, where after the physiological changes of pregnancy cause the value to increase. ${ }^{23,32,33,35}$ Elevated levels of B-type natriuretic peptides (NT-pro-BNP) in nonpregnant patients were associated with complicated disease. ${ }^{36}$ Elevated NT-pro-BNP levels had an OR for death of 8.7 (95\% CI 2.8-27) and an OR for complicated clinical outcome of 6.8 (95\% CI 4.4-10). ${ }^{36}$ The combination of elevated D-dimer and NT-Pro-BNP levels has been described as an aid in the diagnosis of PE in pregnancy. ${ }^{37}$

Special imaging. Ventilation perfusion scans (VQ scan). VQ scans can be used in pregnancy to aid in the diagnosis but $73.5 \%$ are reported as normal compared with 10 to $30 \%$ of nonpregnant patients. In one study, only $2 \%$ had a high probability scan result. ${ }^{23,25,29-31,33,38-41}$ The follow-up of untreated women after a normal or low probability scan showed no adverse effects. A normal scan will exclude PE and a high probability scan has a positive predictive value of 85 to $90 \%$ but the sensitivity is only $41 \%$ with a specificity of $97 \% .{ }^{23,30}$ The radiation with VQ scanning is estimated to be $100-370 \mu \mathrm{Gy}$, ${ }^{38}$ which is far less radiation on the maternal breast than with spiral $\mathrm{CT}^{33,35}$ To reduce foetal exposure to radiation, only a perfusion scan is performed. ${ }^{32}$ Other authors feel that most exposure is due to the perfusion scan and therefore there is no need to reduce the amount of the ventilation scan. They support the idea that a ventilation scan is not indicated in a patient with a normal perfusion scan and that this is enough to rule out a PE. ${ }^{25,31,38}$ When the pretest clinical probability of a PE is low the finding of a low probability VQ scan is as reliable as a CT angiogram to exclude a pulmonary embolism, and a high clinical probability and a high probability VQ scan is enough evidence to start treatment with a positive predictive value of 85 to $90 \% .{ }^{23,31,35,38}$ If the clinical probability of PE is high and the VQ scan is not diagnostic further investigations should be done to exclude the diagnosis. ${ }^{23,25,29}$

Venous compression duplex ultrasound (VCUS). VCUS is indicated in patients with a high clinical probability of PE with other risk factors such as leg symptoms $29,30,33,38,40$ and it is especially useful in women less than 20 weeks gestation. The sensitivity is $95 \%$ with specificity of $96 \%$ to diagnose proximal DVT. $^{23}$ In patients without suspected deep venous thrombosis and a high clinical suspicion, $10 \%$ will have confirmed PE with negative VCUS. ${ }^{31,42}$ If the VCUS is positive, treatment can be initiated for PE as long as the test was correctly performed. ${ }^{26,38}$ Serial follow-up VCUS can also be valuable in excluding patients with $\mathrm{PE}^{30}$

Computer tomography pulmonary angiography (CTPA). CTPA can be used to help with the diagnosis of $\mathrm{PE}$ in pregnancy. ${ }^{25,32}$ The radiation risk for the foetus is less than with a VQ scan. ${ }^{23}$ Exposure of the foetus can be reduced by ${ }^{43}$ using a lead apron with a thickness of $0.7 \mathrm{~mm}$ that covers the patient. A lesser thickness can be used over the back of the patient, maximising the distance between the edge of the scan and the foetus. The risk of CTPA radiation is actually to the mother. ${ }^{33,37,42}$ Each breast is exposed to approximately 20-50 mGy during a chest CT, which increases a woman's lifetime risk to develop breast cancer by 13 to $14 \%$ (1:143 in a 20 -year-old patient). ${ }^{33,35}$ The advantage of CTPA is that it has a sensitivity and specificity between 94 and $100 \%$ in nonpregnant patients and evaluation of available data showed the negative likelihood ratio of pulmonary embolism after a negative scan was 0.07 and the negative predictive value was $99.1 \%{ }^{23}$ The main problem in pregnancy is that the percentage of non-diagnostic tests is higher than in nonpregnant patients (5-10\%). ${ }^{31}$

CTPA is the best modality for diagnosing emboli in the main, lobar and segmental arteries. With advances in the development of multiple thin slices the diagnosis of emboli in the subsegmental arteries has improved to such a level that the test is comparable in sensitivity to pulmonary angiography with greater accuracy in diagnosing distal emboli than a VQ scan. ${ }^{23,31}$ The added advantage of CTPA over a VQ scan is the ability to identify other causes of the patient's clinical picture and the test is more frequently available after hours. ${ }^{23}$

Pulmonary angiogram. This was the gold standard for the diagnosis of PE but is associated with $0.5 \%$ mortality and $5 \%$ morbidity. There is also higher radiation of the foetus and breasts than with CTPA. ${ }^{23,31}$ As CTPA has comparable sensitivity and specificity, pulmonary angiography is no longer part of the routine work up of a pregnant patient with suspected PE. ${ }^{23,31}$

Other imaging modalities. MRI angiography with gadolinium contrast has been evaluated in nonpregnant patients but there is no experience in pregnant patients and it is not 
recommended. ${ }^{23,25,31,42}$ Echocardiography indicating right ventricular strain can aid in the diagnosis. In some cases emboli can be seen passing through the right heart. ${ }^{25}$

\section{Management}

Non-life-threatening PE. Management of a patient with PE depends on the clinical picture. In the case of a non-life-threatening PE, the patient should be started on low-molecular-weight heparin (LMWH) or unfractionated heparin (UFH). ${ }^{23,25,30,32,33}$ If UFH is used, the activated partial thromboplastin time (aPTT) should be 1.5 to 2.5 the average laboratory time taken 6 hours after the administration of the drug and the therapeutic level should be achieved in 24 hours to reduce the risk of recurrent emboli. ${ }^{23,25,32,33}$ The American College of Chest Physicians, based on grade 1A evidence, and the Royal College of Obstetricians and Gynaecologists recommend management with LMWH with the advantage that platelet monitoring is not indicated. ${ }^{23,25,32,33}$ The patient should be started on one of the following regimens: $1 \mathrm{mg} / \mathrm{kg}$ enoxaparin 12 hourly, $90 \mathrm{U} / \mathrm{kg}$ deltaparin 12 hourly or $90 \mathrm{U} / \mathrm{kg}$ tinzaparin 12 hourly or $175 \mathrm{U} / \mathrm{kg}$ daily. ${ }^{32,33}$ There is controversy regarding anti-Xa level monitoring in pregnant patients receiving LMWH. Some authors monitor the levels in patients less than $45 \mathrm{~kg}$ or more than $90 \mathrm{~kg}^{32,33}$ while others measure it monthly. ${ }^{23,25}$ The level should be 0.4 to $1.2 \mathrm{U} / \mathrm{ml} 3$ to 4 hours after injection. $23,32,33$ Some authors recommend unfractionated heparin in the following circumstances ${ }^{23,32}$ :

- Massive pulmonary embolism

- Patients at increased risk of bleeding

- Close to delivery

The reason for this is UFH has a shorter half-life and can be easily reversed with protamine sulphate. ${ }^{23}$ UFH can cause osteopenia and thrombocytopenia that occurs immediately or 5 to 14 days after initiating treatment. Patients on UFH should have their platelet count checked on initiation, day 3 , daily on days 5 to 14 and then monthly. ${ }^{23}$ Neither of these side effects is associated with LMWH. ${ }^{23}$ The treatment with therapeutic dosage should continue for 20 weeks and if the patient is then still pregnant or in the puerperium, prophylactic dosage should continue for until 6 weeks postdelivery. ${ }^{23}$ In the Royal College Guidelines it is stated that treatment at therapeutic levels should continue until 6 weeks postpartum. ${ }^{33}$ If the patient had a vaginal delivery, treatment should restart 3 to 6 hours after delivery, and if she had a caesarean section it should be restarted 6 to 8 hours postdelivery. ${ }^{23}$ If there is an underlying condition that places the patient at high risk she should stay on therapeutic dosage until 6 weeks postdelivery. ${ }^{23}$ Warfarin can be started in the puerperium for the management of these women and then the INR should be maintained between 2 and $3 .^{23,32}$

Massive pulmonary embolism. The ABC of management of a patient with a collapse should be followed. The patient should be transferred to an Intensive Care Unit, or if not available to a high care unit, with multi-disciplinary team management. ${ }^{23,33}$ In the case of a massive PE she should be started on intravenous UFH and she should be considered for thrombolysis or even embolectomy. ${ }^{32,33,44}$ In some women a resistance to heparin develops and the APTT does not change. To prevent overdose the activated Xa levels can be measured. ${ }^{33}$ In cases of a massive pulmonary embolism, the Royal College suggests the following treatment protocol with $\mathrm{UFH}^{33}$ :

- Loading dose should be $80 \mathrm{U} / \mathrm{kg}$.

- Administer continuous intravenous infusion of $18 \mathrm{U} / \mathrm{kg} / \mathrm{hour}$.

- If she had thrombolysis the loading dose can be omitted.

- Check the APTT 6 hours after the loading dose, 6 hours after any drug change and the daily when APTT is between 1.5 and 2.5 the normal.

- The Royal College Guidelines have protocols how to adjust the UH to achieve the required APTT level if it is not correct. 


\section{Acute coronary syndrome}

The incidence of acute coronary syndrome in pregnancy ranges from 1 in 10,000 to 1 in 35,700 pregnancies. ${ }^{45-47}$ In the United Kingdom, the number of women dying from myocardial infarction due to ischemic heart disease is increasing. ${ }^{1}$ Poor life-style habits, smoking, obesity, hypertension as well as delaying pregnancy to a more advanced age are associated with the development of myocardial infarction. ${ }^{1}$

\section{Patophysiology}

A study of coronary artery morphology on pregnant and postpartum women found the following ${ }^{46}$ :

- Coronary atherosclerosis (with or without thrombus) was found in $43 \%$ of patients.

- Thrombus without evidence of atherosclerosis was present in $21 \%$.

- Coronary dissection was present in $16 \%$.

- Normal coronary arteries were seen in $16 \%$ of the women.

The discovery of thrombus formation in the absence of atherosclerosis in $21 \%$ of patients is believed to be the result of a transient coronary spasm causing an acute coronary thrombosis. ${ }^{46}$ The hypercoagulable state of pregnancy promotes thrombus formation. Patients with pregnancies complicated by pre-eclampsia, uterine hypoperfusion and those treated with ergot alkaloids are at an increased risk of developing coronary vasospasm. ${ }^{46}$ In pre-eclampsia the vasospasm may be caused by endothelial dysfunction and enhanced vascular reactivity to angiotensin II, while increased renin release and angiotensin production cause coronary vasospasm in patients with decreased uterine perfusion.

\section{Risk factors}

Risk factors for the development of an acute coronary syndrome include $e^{48}$ :

- Maternal age above 35 years

- African American race

- Smoking

- Medical conditions such as thrombophilia, hypertension, diabetes mellitus and anaemia

Pregnancy-related risk factors are ${ }^{48}$ :

- Pre-eclampsia

- Transfusion

- Postpartum infection

- Fluid and electrolyte imbalance

Acute coronary syndrome occurs most frequently in the third trimester in multigravidas and is most commonly located in the anterior wall. ${ }^{46}$

\section{Diagnosis}

The diagnosis may be delayed due to the low index of suspicion of the condition amongst clinicians, and the typical symptoms such as chest pain, dyspnoea and nausea may mimic the common discomforts of pregnancy such as reflux oesophagitis, hiatus hernia and uterine contractions. ${ }^{49}$

The diagnosis is confirmed by finding typical electrocardiographic (ECG) changes and specific cardiac markers (troponin $\mathrm{T}$ or $\mathrm{I}$ ). Myocardial function assessment by echocardiography is recommended.

\section{Management}

Once a diagnosis is made, the patient should receive a treatment modality that would ensure safety of both mother and baby. The recommended treatment strategy based on limited data from observational studies and case reports is immediate coronary angiography and percutaneous coronary 
intervention as a re-vascularisation method (with or without stenting). ${ }^{50}$ In pregnancy the radial approach is preferred to the femoral approach as this reduces the radiation exposure to the foetus. The foetus is most sensitive to radiation between 8 and 15 weeks gestation. ${ }^{45}$ If coronary stent placement is required the preferred choice is a bare metal stent rather than a drug-eluting stent as a shorter duration of treatment with a platelet aggregation inhibitor such as clopidrogel is needed. Although there are reports of the use of clopidrogel in pregnancy, there are few data on its effect on the foetus. Therefore, an alternative may be balloon dilatation without stenting. ${ }^{45}$ There is no clinical evidence for fibrinolytic therapy as a reperfusion strategy in pregnancy and is best avoided as the risk of haemorrhage outweighs the possible benefit of treatment. Low molecular weight heparin should be used to prevent further thrombosis. Morphine may be used for symptomatic pain relief, but may cause respiratory depression in the neonate if given just before delivery. Although oral and intravenous nitrates have been used for the treatment of hypertension and myocardial ischemia there is limited information on the safety of this drug. ${ }^{45}$

After the initial reperfusion strategy there are limited therapeutic agents that can be used to reduce the risk of recurrence and ventricular re-modelling. Cardio-specific beta-blockers, low dose aspirin, furosemide and spironolactone, are safe to use in pregnancy. The calcium antagonist, diltiazem, is teratogenic. ${ }^{3}$ Although angiotensin-converting inhibitors are standard therapy in the nonpregnant individual they are toxic to the foetus and the following complications have been reported ${ }^{51}$ :

- Neonatal renal failure

- Oligohydramnios

- Intra-uterine growth restriction

- Hypoplasia of skull bones

Labour and delivery should ideally be delayed for at least 2 weeks after the infarct to allow healing to take place. Both vaginal delivery and an elective caesarean section are associated with advantages and disadvantages and there is no convincing support for one method over the other. ${ }^{46}$ The mode of delivery must be guided by the haemodynamic profile of the patient. Patients undergoing vaginal delivery should be nursed in the left lateral position with continuous haemodynamic monitoring and delivery should be assisted to avoid excessive maternal effort. The development of hypertension tachycardia should be avoided. Adequate pain relief is therefore very important. Patients who may still be on dual anti-platelet therapy must be monitored closely for signs of bleeding.

\section{Thrombosed mechanical prosthetic heart valves}

Pregnancy is a hypercoagulable state and patients with prosthetic heart valves have a $3-14 \%$ risk of a thromboembolic event despite optimal anti-coagulation. ${ }^{52}$ Pregnancy poses additional challenges because vitamin $\mathrm{K}$ antagonists cross the placenta and are associated with an increased risk of pregnancy loss, embryopathy and foetal intra-cranial haemorrhage. ${ }^{53}$ Long-term use of unfractionated heparin is associated with osteoporosis and thrombocytopenia.

\section{Classification}

Prosthetic heart valve thrombosis is classified into 4 functional classes according to the New York Heart Association ${ }^{54}$ :

- Class I and II are non-obstructive forms and the diagnosis is usually made by an incidental finding on echocardiogram.

- Classes III and IV are obstructive forms and are often associated with cardiogenic shock, haemodynamic change and cerebral or peripheral embolism.

\section{Diagnosis}

Auscultation may reveal a decrease or disappearance of the prosthetic valve sound and this may be associated with a new murmur that was not present previously. Confirmation of diagnosis is by transthoracic echocardiography and fluoroscopy with appropriate abdominal shielding. 


\section{Management}

Management options for the treatment of prosthetic valve thrombosis in pregnancy are limited and based anecdotal case reports. Acute collapse in the presenting patient is most probably due to a large thrombosed obstructed clot. This is a medical emergency and all decision making in terms of therapy should be based on maternal outcome. In nonpregnant patients, surgery or thrombolytics are the first line therapy in the acute situation. There are reports of use of fibrinolytics and surgery in pregnancy as therapeutic options but both strategies are associated with increased foetal loss rates. ${ }^{55} \mathrm{~A}$ review of 172 patients receiving thrombolytic therapy for various indications showed a foetal loss rate of $5.8 \%$ and bleeding risk of $8.1 \%{ }^{55}$

Patients with prosthetic valves should be educated about the increased risk for thrombosis in pregnancy and the need to maintain therapeutic anti-coagulation.

\section{Aortic dissection}

There were nine maternal deaths due to aortic dissection in the United Kingdom between 2003 and 2005 and this number has increased from the previous report. ${ }^{1}$ Fifty percent of aortic dissections in women under 40 years of age are pregnancy-related. ${ }^{56}$ In pregnancy the most common site is the proximal aorta, and aortic rupture occurs most frequently in the third trimester or during the first stage of labour. ${ }^{57}$

\section{Patophysiology}

Aortic dissection begins with a tear in the aortic intima and the inner layer of the aortic media. This allows blood to enter and split the medial layer, thus creating a true and false lumen separated by an intimal flap.

Both inherited and acquired conditions predispose to the development of aortic dissection. Systemic hypertension is the most important predisposing factor according to the International Registry of Aortic Dissection (IRAD) ${ }^{58}$ Hypertension may result in a spontaneous rupture of the aortic vasa vasorum. This causes an intramural haemorrhage that weakens the medial layer and leads to the development of an intimal tear. The hypertension contributes to an additional mechanical strain on the aortic wall. ${ }^{59}$ The intimal tear then spreads to the medial layer and this is aggravated in patients with medial degeneration and weakening.

Other acquired causes are:

- Direct trauma

- Iatrogenic retrograde dissection from a catheter-related aortic intimal injury

- Previous valvular, aortic or coronary bypass surgery

The use of cocaine results in a cathecolamine surge and this causes a sudden increase in aortic wall stress that may lead to the development of an aortic dissection.

Inherited conditions also associated with an increased risk include ${ }^{59}$ :

- Marfan's syndrome

- Ehlers-Danlos syndrome

- Bicuspid aortic valve

- Co-arctation of the aorta

In these conditions dissection is caused by cystic medial necrosis or degeneration. ${ }^{59}$

\section{Classification}

Aortic dissections are classified according to their anatomic location. The Stanford type A dissection involves the ascending aorta and the type B dissection involves the aorta distal to the origin of the left subclavian artery. 


\section{Diagnosis}

The most common manifestation of aortic dissection is the abrupt onset of severe back or chest pain that is described as stabbing, ripping or tearing. ${ }^{60}$ However, a study over a 27 -year period showed that the diagnosis is missed in $85 \%$ of cases. ${ }^{61}$ The pain often migrates along the path of the dissection. Syncope may be present and is more common in patients with type A dissections. ${ }^{59}$ Seventy percent of patients with type $\mathrm{B}$ and $36 \%$ of patients with type A dissections present with hypertension. ${ }^{59}$ Hypotension occurs in the advanced stages of the disease and is due to cardiac tamponade, aortic rupture or severe aortic regurgitation.

Other important clinical findings are:

- A diastolic murmur due to aortic regurgitation

- Pulse deficits

- Signs of congestive heart failure

- Focal neurological deficits

The diagnosis is confirmed by special investigations such as echocardiography, computed tomography or magnetic resonance imaging.

\section{Management}

The recommended management principles are based on a 12-year review of aortic dissection in pregnancy by Zeebregts et al.. ${ }^{62}$ Type A dissections are surgically corrected with the aim of preserving 2 lives:

- Before 28 weeks, the aorta is repaired without delivery of the foetus.

- After 32 weeks the foetus is delivered by caesarean section and the aortic repair is done at the same operation.

- Between 28 and 32 weeks the decision to deliver the foetus will be influenced by the foetal condition.

Cardiovascular surgery in pregnancy is associated with a $20-30 \%$ maternal mortality and 2-6\% foetal mortality rates. ${ }^{60}$ Type $\mathrm{B}$ dissections are treated medically with the aim of controlling the blood pressure.

Patients must be managed in an intensive care unit and intubation and mechanical ventilation is needed for unstable patients. Morphine is used for pain and to reduce the sympathetic stimulation. Intravenous beta-blockers are the first line drugs for controlling blood pressure. The aim is to reduce the systolic blood pressure to $110 \mathrm{mmHg} .{ }^{59}$ If the blood pressure is still uncontrolled after betablockade, additional vasodilator treatment in the form of sodium nitroprusside is added. It is important to look for signs of blood loss, heart failure or pericardial effusion in normotensive patients.

Acute aortic dissection in pregnancy is rare but is associated with high mortality rates. It is important that clinicians consider this diagnosis if a patient presents with the typical symptoms.

\section{Cerebrovascular incidents in pregnancy}

Approximately 26-34 per 100,000 pregnancies are complicated by stroke. ${ }^{63,64}$ Physiological changes in pregnancy such as the increased coagulability, venous stasis and fluctuations in blood pressure are predisposing factors. Forty percent of strokes occur during delivery and $50 \%$ in the postpartum period. ${ }^{64}$ Stroke in pregnancy may be ischemic or hemorrhagic.

\section{Risk factors}

The risk for stroke is increased in the following women:

- Aged 35 years and older

- African-American race 
- Medical conditions such as migraine, thrombophilia, systemic lupus erythematosus, cardiac disease, sickle cell disease, hypertension and thrombocytopenia

Pregnancy-related risk factors include ${ }^{64}$ :

- Pre-eclampsia

- Gestational hypertension

- Blood transfusion

- Postpartum infection

\section{Patophysiology}

Ischemic stroke may be caused by vascular thrombosis, embolism, vasospasm or a sudden severe hypotensive incident. Cardiac causes of cerebral emboli include rheumatic heart disease, mitral valve prolapse, atrial septal defects, patent foramen ovale and bacterial endocarditis. Arterial diseases associated with embolism are artherosclerosis, Takayashu's disease, Moya-moya disease and arterial dissection. ${ }^{65}$ Cerebral haemorrhage may be either intracerebral or subarachnoid. Intracerebral haemorrhage is most often due to severe hypertension, coagulopathy or vascular malformations, while a subarachnoid haemorrhage is usually the result of a ruptured aneurysm or arteriovenous malformation. ${ }^{65}$

\section{Clinical presentation}

A stroke must be suspected when a patient presents with an acute onset of focal neurological signs. In addition, the patient may be confused with a depressed level of consciousness and complain of a headache. Seizures may be a secondary manifestation. ${ }^{66}$ It is important to distinguish stroke from conditions that present with similar symptoms such as migraine and focal seizures.

\section{Diagnosis}

The diagnostic approach in pregnancy is no different than in the nonpregnant patient. Either a non-contrast computed tomography (CT) or magnetic resonance imaging (MRI) is an essential diagnostic tool. CT scans are usually more widely available, are quicker and less expensive than MRI. A CT scan performed with abdominal screening results in $2 \mathrm{mrad}$ exposure to the foetus and this is lower than accepted maximum threshold dose. ${ }^{65,66}$ There are no short-term risks to the foetus after MRI scanning although long-term effects are still unknown. ${ }^{65}$ Imaging should help in determining whether the stroke is ischemic or hemorrhagic, however, an early infarction is sometimes missed on CT scan. ${ }^{67}$

Echocardiography and Doppler studies are indicated if cardiac or carotid pathology is suspected. A lumbar puncture may be helpful in diagnosing a small subarachnoid bleed that is not always visible on CT or MRI. A lumbar puncture will also exclude infection.

\section{Management}

A pregnant patient with a stroke should be managed by a multi-disciplinary team. The principles of treatment are to protect the remaining brain tissue and prevent further complications. Antihypertensive therapy is indicated if the mean arterial blood pressure is greater than $125 \mathrm{mmHg}$. Ischemic stroke is generally treated with anticoagulants, anti-platelet agents or thrombolytic therapy. Warfarin is teratogenic and should be avoided in the first trimester. Heparin does not cross the placenta but it is associated with the complications of osteoporosis and thrombocytopenia. Unfractionated heparin is advised around the time of delivery and it should be withheld for 6 hours after vaginal delivery and 12 hours after caesarean section due to the risk of bleeding. Single transient ischemic attacks may be treated with low dose aspirin therapy but if attacks recur therapeutic doses of heparin must be started. ${ }^{68}$ Thrombolytic therapy has been proven to be effective for the treatment of acute ischemic arterial stroke in nonpregnant subjects. ${ }^{66}$ However, as mentioned earlier, the use of thrombolytic agents in pregnancy has been associated with hemorrhagic complications in the mother and foetus and is not recommended. 
Hemorrhagic strokes caused by aterio-venous malformations are managed according to neurosurgical principles. Surgical intervention is not needed if both the bleeding and patient are stable. ${ }^{65}$ Surgical decompression is indicated if the brainstem is compromised.

The timing and mode of delivery depends on the maternal condition and gestational age of the foetus. Delivery is indicated if the gestational age is above 34 weeks, or in the case of non-reassuring foetal status or an unstable maternal condition. ${ }^{65}$ Below 26 weeks gestation, management should be guided by maternal diagnosis and condition. Between 26 and 34 weeks, an attempt should be made to administer steroids for foetal lung maturity if the maternal condition is stable. If the mother remains stable, the foetus may be delivered at term. There are no randomised trials comparing elective caesarean section to an assisted vaginal delivery and regional anaesthesia, therefore caesarean delivery should be reserved for obstetric indications.

The risk of recurrence in subsequent pregnancies is about $1 \%{ }^{69}$ but is greater (27\%) for women with untreated risk factors such as thrombophilia. ${ }^{70}$

\section{Problems related to the use of general anaesthesia}

\section{Failed intubation}

Failed intubation is the number 1 cause of anaesthesia-related maternal deaths in the world today. ${ }^{71}$ In the USA the incidence of failed intubation in obstetric patients is $1: 280$, compared with $1: 2,230$ in non-obstetric patients. ${ }^{72}$ The changed anatomy and physiology of pregnancy contributes largely to the fact that pregnant patients often present with extreme difficulties in airway management. These changes and their effects are ${ }^{73}$ :

- Capillary engorgement leads to airway oedema

- Gravid uterus causes cephalad displacement of the diaphragm and decreased functional residual capacity

- Changed angle of the gastro-oesophageal junction and secretion of muscle-relaxing hormones cause delayed gastric emptying and increased risk of aspiration

- Excessive weight gain and breast enlargement cause difficult intubation

- Increased oxygen consumption leads to increased oxygen requirements

Although it is absolutely essential for the anaesthetist to do a thorough evaluation of the obstetric patient's airway before any type of anaesthesia is administered, always being prepared for the possibility of having to deal with a difficult airway is the best way to address this problem. If at all possible, a regional anaesthetic should be used if a difficult airway is suspected. If this is impossible, adequate positioning of the patient, the use of smaller than usual laryngoscope blades, the placement of a laryngeal airway mask or similar type device, which does not necessitate endotracheal intubation, or even awake fibre-optic intubation techniques may be employed to assure a safe airway. If none of the latter is available, mask ventilation with continuous, effective cricoid pressure may be the only alternative when the patient cannot be awakened.

\section{Anaphylaxis}

The incidence of anaphylaxis due to anaesthetic agents is extremely low. It is therefore not warranted to routinely investigate patients who are asthmatic or have a history of allergy or atopy preoperatively. However, all patients with a history of a previous reaction to an anaesthetic agent should receive skin testing before administration of such a drug, or variants thereof. Special efforts should also be made to elicit a history of possible latex allergy. ${ }^{74}$

\section{Problems relating to regional or local anaesthetic agents}

\section{Central nervous system toxicity}

Although the newer local anaesthetic drugs such as ropivacaine and levobupivacaine have a better safety profile than bupivacaine, all of these drugs, if used in higher than normal doses or if 
inadvertently injected into the intrathecal space, may cause progressive depression of the CNS and ultimately coma and seizures. It is therefore important to always use these drugs in incremental dosing and always to give a test dose before administration of the full dose, in order to identify individuals who may be hypersensitive to them.

\section{Cardiac arrest}

Although regional neuraxial anaesthesia is generally considered the safer alternative to general anaesthesia, it has been well described that cardiac arrest can occur in seemingly healthy individuals receiving regional blocks. Specific risk factors for cardiac arrest under these circumstances have been identified $^{75}$ :

- Baseline heart rate $<50 \mathrm{bpm}$

- ASA physical status 1

- Use of a beta-blocker

- Sensory blockade above the level of T6

- Age $<50$ years

- A prolonged PR interval

The mechanism proposed for this phenomenon is the exaggerated effect of sympathetic blockade on central venous pressure and vagal tone in selected individuals. It appears as if the unique physiology of pregnancy, with a decreased parasympathetic tone and higher baseline heart rates, protect the pregnant patient from cardiac arrest related to regional anaesthesia to some extent. However, due to the increase in the number of pregnant patients receiving these regional blocks in labour, it is likely that the number of cardiac arrests in labour wards will also increase in the next few years.

The most important measure to prevent this disastrous event is to give the patient an adequate fluid preload before administering the block and to maintain good hydration status thereafter.

It is also helpful to know that the CNS side effects of these local agents (such as tongue numbness, dizziness and audiovisual disturbances) occur at much lower blood levels than cardiovascular effects, and these may serve as an early warning sign to discontinue the use of the drug and to adequately hydrate such patients. ${ }^{4}$

Bupivacaine is much more cardiotoxic than lidocaine, and it also appears that pregnant patients are especially sensitive to the cardiotoxicity of bupivacaine.

It is therefore wise, in instances where cost permits it, to avoid bupivacaine entirely in pregnant patients.

High spinal/epidural block

The main problems with a high block are:

- Lack of preload and an empty heart, leading to severe hypotension and decreased cardiac output

- Paralysis of the respiratory muscles, leading to hypoxia and aspiration

Treatment includes adequate fluid administration, airway management, pressors, left-sided displacement of the uterus and elevation of the legs.

Other causes of collapse in theatre

In cases of sudden seizures or cardiac arrests in theatre patients, the following possible causes, all proven to be hazardous, must be considered as alternatives to direct reactions to anaesthetic agents:

- Amniotic fluid embolism

- Oxytocin administered as an IV bolus ${ }^{76}$

- Air embolism

- Blood transfusion reaction 


\section{Summary}

A good differential diagnosis and a high index of suspicion are crucial in the approach to the pregnant patient with acute collapse. A good history of underlying medical condition may help to recognise some of the cardiac causes of a patient with acute collapse.

Anaesthesia, although safe in the hands of an experienced operator and a healthy patient, is still an unnatural intervention and needs to be approached with the utmost caution in any case. This is even truer for the pregnant patient, where two lives are at stake. Anaesthesia is by no means harmless, and an awareness of its possible complications, together with the know-how to act promptly in such cases, will no doubt save lives. There is a degree of uncertainty regarding the best diagnostic tests for the different conditions. The problem is further aggravated by the lack of or the difficulty of doing wellstructured research on these conditions.

\section{Practice points}

- High index of suspicion in a patient with acute collapse for AFE

- Supportive management of the different affected systems

- If undelivered, perimortem caesarean section within 5 minutes if no response

- D-dimer assessment can only be used in patients with low clinical probability

- VQ Scan or CPTA is the diagnostic method of choice depending on availability

- CPTA radiation risk is more of a concern regarding maternal radiation than foetal radiation

- LMWH is the treatment option of choice

- Know the CNS side effects of the anaesthetic drugs used

- Identify the women who are at risk for a difficult intubation

- Myocardial infarction and aortic dissection must be considered as a possible diagnosis in patients who present with acute chest or epigastric pain

- Therapeutic anticoagulation levels must be maintained in patients with mechanical prosthetic heart valves

- Doing activated Xa levels on women on LMWH is indicated in pregnancy

- Know the risk factors fore cardiac arrest in women with regional anaesthesia

\section{Research agenda}

- The place of D-dimer in diagnosis op PE in pregnancy

- The gold standard for diagnosis PE in pregnancy

- The place of other treatment modalities for AFE and PE in pregnancy

- The safety of clopidrogel in pregnancy

- The safety of fibrinolytic therapy in pregnancy

\section{Acknowledgements}

Thank you to Prof. A.P. MacDonald for his help with the language editing and for Prof. T. Mathivha who helped with the content of the cardiac causes.

\section{References}

1. Pattinson RC (ed.). Saving Mothers: third report on confidential enquiries into maternal deaths in South Africa 2002-2004 Executive summary. Pretoria: Government Printer, 2006. 
2. Confidential Enquiry into Maternal and Child Health. Saving Mothers Lives: reviewing maternal deaths to make motherhood safer - 2003-2005. Executive summary and key recommendations. London: RCOG Press, 2007, www.cemach.org.uk.

3. Lombaard HA \& Pattinson RC. Undelying medical conditions. Best Pract Res Clin Obstet Gynaecol 2008; 22: 847-864.

*4. Stafford I \& Sheffield J. Amniotic fluid embolism. Obstet Gynecol Clin North Am 2007; 34: 545-553.

5. Swayze CR, Barton JR \& Skerman JH. Amniotic Fluid Embolism. S Anest Peri Op Med Pain 2000; 19: 181-187.

6. Banks A \& Levy D. Life-threatening complications of pregnancy: Key issues for anaethetists. Curr Anaesth Crit Care 2006; 17: $163-170$.

7. James CF, Feinglass NG, Menke DM et al. Massive amniotic fluid embolism: diagnosis aided by emergency transesophageal echocardiography. Int J Obstet Aneast 2004; 13: 279-283.

8. Schoening AM. Amniotic fluid embolism: historical perspectives and new possibilities. MCN 2006; 31: 78-83.

9. McDonnel NJ, Chan BO \& Frenley RW. Rapid reversal of critical haemodynamic compromise with nitric oxide in a parturient with amniotic fluid embolism. Int J Obstet Aneast 2007; 16: 269-273.

10. Clark SL, Hankins GD, Dudley DA et al. Amniotic fluid embolism: Analysis of the national registry. Am J Obstet Gynecol 1995; 172: 1159-1167.

11. Tuffnel DJ. Amniotic fluid embolism. Curr Opin Obste Gynecol 2003; 15: 119-122.

*12. O'Shea A \& Eappen S. Amniotic fluid embolism. Int Anesthesiol Clin 2007; 45: 17-28.

13. Aurangzeb I, George L \& Raoof S. Amniotic fluid embolism. Crit Care Clin 2004; 20: 643-650.

14. Kent KJ, Cooper BC, Thomas KW et al. Persumed antepartum amniotic fluid embolism. Obstet Gynecol 2003; 102: $493-495$.

*15. Nelson-Piercy C. Differential diagnosis of medical problems in pregnancy Table 10. In Nelson-Piercy C (ed.). Handbook of Obstetric Medicine. 3rd edn. Oxon: Informa Healthcare, 2006. pp. 313-340.

16. Kane SK. Historical perspective of amniotic fluid embolism. Int Anesthesiol Clin 2005; 43: 99-108.

17. Locksmith GJ. Amniotic fluid embolism. Obstet Gynecol Clin North Am 1999; 26: 435-444.

18. Aguilera LG, Fernandez C, Laza AP et al. Fatal amniotic fluid embolism diagnosed histologically. Acta Aneasthesiol Scand 2002; 46: 334-337.

19. Fletcher SJ \& Parr MJ. Amniotic fluid embolism: a case report and review. Resuscitation 2000; 43: 141-146.

20. Rudisill PT. Amniotic fluid embolism. Crit Care Nurs Clin North Am 2004; 16: 221-225.

21. To MS, Hunt BJ \& Nelson-Piercy C. A negaitive D-Dimer does not exclude venous thromboembolism (VTE) in pregnancy. J Obstet Gynaecol 2008; 28: 222-223.

*22. Knight M, \& on behalf of UKOSS. Antenatal pulmonary embolism: risk factors, management and outcomes. Br J Obstet Gynaecol 2008; 115: 453-461.

23. Rosenburg VA \& Lockwood CJ. Thromboembolism in pregnancy. Obstet Gynecol Clin North Am 2007; 34: 481-500.

24. Sakuma M, Nakamura M, Takahashi T et al. Pulmonary embolism is an important cause of death in young adults. Circ J 2007; 71: 1765-1770.

25. Tapson VF. Acute pulmonary embolism. N Engl J Med 2008; 358: 1037-1052.

26. Fedullo PF \& Tapson VF. The evaluation of suspected pulmonary embolism. N Engl J Med 2003; 349: 1247-1256.

27. Fennerty T. The patient with possible pulmonary embolism. Acute Care 2008: 20-24.

28. Voke J, Keidan J, Pavord S et al. British Society for Haematology Obstetric Haematology Group. Br J Haematol 2007; 139: 545-558.

29. Mallick S \& Petkova D. Investigating suspected pulmonary embolism during pregnancy. Respir Med 2006; 10: $1682-1687$.

30. Rodger MA, Walker M \& Wells PS. Diagnosis and treatment of venous thromboembolism in pregnancy. Best Pract Res Clin Haem 2003; 16: 279-296.

31. Scarsbrook AF, Evans AL, Owen AR et al. Diagnosis of suspected venous thromboembolic disease in pregnancy. Clin Radiol 2006; 61: 1-12.

*32. Nelson-Piercy C. Thromboembolic disease. In Nelson-Piercy C (ed.). Handbook of obstetric medicine. 3rd edn. Oxon: Informa Healthcare, 2006. pp. 45-63.

33. Greer IA \& Thomson AJ. Thromboembolic disease in pregnancy and the puerperium: acute management. RCOG Green-top Guideline no 28, www.rcog.org.uk; February 2007.1-17.

34. Parent F, Maître S, Meyer G et al. Diagnostic value of D-Dimer in patients with suspected pulmonary embolism: results from a multicentre outcome study. Thromb Res 2007; 120: 195-200.

*35. Stein PD, Sostman HD, Bounameaux H et al. Challenges in the diagnosis acute pulmonary embolism. Am J Med 2008; 121: 565-571.

36. Klok FA, Mos ICM \& Huisman MV. BNP levels in the prediction of adverse pregnancy outcome in patients with pulmonary embolism: a meta-analysis. Am J Respir Crit Care Med 2008; 15: 425-430.

37. Franz MB, Guenthner-Biller MM, Kainer F et al. Pro-B-type natriuretic peptide as a marker of pulmonary embolism in pregnancy: a case report. Arc Gynecol Obstet 2008; 277: 439-440.

38. Matthews S. Short communication: imaging pulmonary embolism in pregnancy: what is the most appropriate protocol? Br J Radiol 2006; 79: 441-444.

39. Rodger M \& Wells PS. Diagnosis of pulmonary embolism. Thromb Res 2001; 103: v225-v238.

40. Chan WS, Ray JG, Murray S et al. Suspected pulmonary embolism in pregnancy. Clinical presentation, results of lung scanning and subsequent maternal and paediatric outcomes. Arch Intern Med 2002; 162: 1170-1175.

41. Dresang LT, Fontaine P, Leeman L et al. Venous thromboembolism during pregnancy. Am Fam Physician 2008; 77: 1709-1716.

*42. Patel SJ, Reede DL, Katz DS et al. Imaging in pregnant patient for nonobstetric conditions: Algorithms and radiation dose considerations. Radiographics 2007: 1705-1722.

43. Kennedy EV, Iball GR \& Brettle DS. Investigation into the effect of lead shielding for fetal dose reduction in CT pulmonary angiography. Br J Radiol 2007; 80: 631-638.

44. Taniguchi S, Fukuda I, Minakawa M et al. Emergnecy pulmonary embolectomy during the second trimester of pregnancy: report of a case. Surg Today 2008; 38: 59-61.

*45. Roos-Hesselink JW. Ischaemic heart disease. In Steer PJ, Gatzoulis MA \& Baker P (eds.). Heart disease and pregnancy. RCOG Press, 2006, pp. 243-247.

46. Roth A \& Elkayam U. Acute myocardial infarction associated with pregnancy. Ann Intern Med 1996; 125: 751-762. 
47. Ladner HE, Danielsen B \& Glbert WM. Acute myocardial infarction in pregnancy and the puerperium: a population-based study. Obstet Gynecol 2005; 105: 480-484.

48. James AH, Jamison MG, Biswas MS et al. Acute myocardial infarction in pregnancy: a united states population-based study. Circulation 2006; 113: 1564-1571.

49. Baird SM \& Kennedy B. Myocardial infarction in pregnancy. J Perinat Neonat Nurs 2006; 20: 311-321.

*50. Lange SS \& Jenner M. Myocardial infarction in the obstetric patient. Crit Care Nurs Clin North Am 2004; 16: $211-219$.

51. Butter HS. An overview of the influence of ACE inhibitors on fetal-placental circulation and perinatal development. Mol Cell Biochem 1997; 176: 61-71.

52. Varadarajan P, Isaeff D \& Pai RG. Prosthetic valve thrombosis presenting as an acute embolic myocardial infarction in a pregnant Patient: issues on anticoagulation regimens and thrombolytic therapy. A J CV Ultrasound and allied Tech. Echocardiography 2006; 23: 774-779.

53. Oakley C, Child A, Jung B et al. Expert consensus document on management of cardiovascular diseases during pregnancy. The Task Force on the management of cardiovascular diseases during pregnancy of the European Society of Cardiology. Eur Heart J 2003; 24: 761-781.

*54. Loriga FM, Perez-Lopez H, Santos-Gracia J et al. Prosthetic heart valve thrombosis: Pathogenesis, diagnosis and management. Int J Cardiol 2005; 110: 1-6.

55. Turrentine MA, Braems G \& Ramirez MM. Use of Thrombolytics for the treatment of thromboembolic disease during pregnancy. Obstet Gynecol Surv 1995; 50: 534-541.

56. Eagle KA \& de sanctis RW. Disease of the aorta. In Braunwald E (ed.). Heart disease. Philadelphia: W.B. Saunders, 1992, pp. 1528-1557.

57. Khan AK \& Nair CK. Clinical, diagnostic and management perspectives of aortic dissection. Chest 2002; 122: 311-328.

58. Hagan PG, Nienaber CA, Isselbacher EM et al. The International Registry of Acute Aortic Dissection (IRAD): new insights into an old disease. JAMA 2000; 283: 897-903.

59. Kamalakannan D, Rosman HS \& Eagle KA. Acute aortic dissection. Crit Care Clin 2007; 23: 779-800.

60. Lewis S, Ryder I \& Lovell AT. Peripartum presentation of an acute aortic dissection. Br J Anaesth 2005; 94: 496-499.

61. Mezaros I, Morocz J, Szlavi J et al. Epidemiology and clinicopathology of aortic dissection. A population-based longitudinal study over 27 years. Chest 2000; 117: 1271-1278.

62. Zeebregts CJ, Schepens MA, Hameeteman TM et al. Acute aortic dissection complicating pregnancy. Ann Thorac Surg 1997; 64: $1345-1348$.

63. Jaigobin C \& Silver FL. Stroke and pregnancy. Stroke 2000; 31: 2948-2951.

64. James $\mathrm{AH}$, Bushnell $\mathrm{CD}$, Jamison MG et al. Incidence and risk factors for stroke in pregnancy and puerperium. Obstet Gynecol 2005; 106: 509-516.

65. Sibai BM \& Coppage KH. Diagnosis and management with stroke during pregnancy/postpartum. Clin Perinatol 2004; 31: 853-868.

66. Feske SK. Stroke in pregnancy. Semin Neurol 2007; 27: 442-452.

67. Kutcher J, Lee MJ \& Hickenbottom S. Cerebrovascular disorders complicating pregnancy. UpTo Date 2007; 16(7): 1-9.

68. Wiebers DO. Ischemic cerebrovascular complications of pregnancy. Arch Neurol 1985; 42: 1106-1113.

69. Lamy C, Hamon JB, Coste J et al. Ischemic stroke in young women. Neurology 2000; 55: 269-274.

70. Soriano D, Carp H, Seidman DS et al. Management and outcome of pregnancy in women with thrombophylic disorders and past cerebrovascular events. Acta Obstet Gynecol Scand 2002; 81: 204-207.

71. Mhyre JM, Riesner MN, Polley LS et al. A series of Anaesthesia-related Maternal Deaths in Michigan, 1985-2003. Aneasth 2007; 106: 1096-1104.

72. Hawkins JL. Anaesthesia-related maternal mortality. Clin Obstet Gynecol 2003; 46(3): 679-687.

73. Munnur U, de Boisblanc B \& Suresh MS. Airway problems in pregnancy. Crit Care Med 2005; 33(Suppl. 10): S259-S268.

74. Chorn R. Complications of regional anaesthesia. Semin Anesth 1996; 15(2): 132-139.

75. Pan PH, Moore CH \& Ross VH. Severe maternal bradicardia and asystole after combined spinal-epidural labor analgesia in a morbidly obese parturient. J Clin Anesth 2004; 16: 461-464.

76. Pinder AJ, Dresner M, Calow C et al. Haemodynamic changes caused by oxytocin during Caesarean Section under spinal anaesthesia. Int J Obst Anaesth 2002; 11: 156-159. 